\title{
A practical guide to photoacoustic tomography in the life sciences
}

\author{
Lihong V Wang \& Junjie Yao
}

The life sciences can benefit greatly from imaging technologies that connect microscopic discoveries with macroscopic observations. One technology uniquely positioned to provide such benefits is photoacoustic tomography (PAT), a sensitive modality for imaging optical absorption contrast over a range of spatial scales at high speed. In PAT, endogenous contrast reveals a tissue's anatomical, functional, metabolic, and histologic properties, and exogenous contrast provides molecular and cellular specificity. The spatial scale of PAT covers organelles, cells, tissues, organs, and small animals. Consequently, PAT is complementary to other imaging modalities in contrast mechanism, penetration, spatial resolution, and temporal resolution. We review the fundamentals of PAT and provide practical guidelines for matching PAT systems with research needs. We also summarize the most promising biomedical applications of PAT, discuss related challenges, and envision PAT's potential to lead to further breakthroughs.

By providing a comprehensive illustration of life from its molecular to its anatomical aspects, modern biomedical imaging has revolutionized the life sciences. Imaging technologies have been used through history to peer into complex biological systems in ever more informative ways, achieving finer spatial resolution, richer contrast, higher imaging speed, deeper penetration, and greater detection sensitivity. At the macroscopic scale, a number of methods, including magnetic resonance imaging, X-ray computed tomography, and ultrasound imaging, achieve excellent penetration for anatomical imaging. Positron emission tomography and single-photon emission computed tomography achieve deep penetration and exhibit high sensitivity to radioactively labeled molecular probes. At the microscopic scale, optical microscopy can detail biological phenomena with subcellular and suborganelle resolutions at superficial depths. However, the different imaging-contrast mechanisms and length scales of these imaging tools have hindered correlative multiscale studies of biological problems. It is thus imperative to build a continuum from microscopic to macroscopic imaging in the life sciences.
In the last decade, PAT (also referred to as optoacoustic or thermoacoustic tomography) has proven capable of multiscale imaging with a consistent contrast mechanism; thus, it is well situated to bridge the microscopic and macroscopic domains in the life sciences. PAT is a hybrid imaging modality that acoustically detects optical absorption contrast via the photoacoustic (PA) effect, a physical phenomenon that converts absorbed optical energy into acoustic energy ${ }^{1}$. The combination of optical excitation with ultrasonic detection offers three advantages. (1) PAT is inherently suited for functional, metabolic, and histologic imaging through endogenous contrast and for molecular and cellular imaging through exogenous contrast. (2) Because biological tissue is orders of magnitude more transparent to sound than to light, in terms of scattering mean free path, PAT provides far greater penetration with a scalable spatial resolution than does optical microscopy. (3) PAT is complementary to and compatible with other imaging modalities, especially optical imaging and ultrasound imaging.

It took more than a century for photoacoustics to evolve from a known physical phenomenon to a

Optical Imaging Laboratory, Department of Biomedical Engineering, Washington University in St. Louis, St. Louis, Missouri, USA. Correspondence should be addressed to L.V.W. (lhwang@wustl.edu).

RECEIVED 1 FEBRUARY; ACCEPTED 20 JUNE; PUBLISHED ONLINE 28 JULY 2016; DOI:10.1038/NMETH.3925 
Figure 1 | Principles of photoacoustic tomography (PAT). (a) Jablonski diagram, illustrating the photon energy transfer in onephoton fluorescence microscopy, two-photon fluorescence microscopy, and PAT. The most common electronic absorption in the visible and ultraviolet spectral regions is shown. (b) Imaging principle of PAT.

valuable biomedical imaging modality. Although the PA effect was first reported by Bell in 1880 with the invention of the photophone $^{1}$, 100 years passed before Bowen proposed to use this phenomenon for imaging by excitation using ionizing radiation (e.g., high-energy electrons and $\mathrm{X}$-ray photons) or nonionizing radiation (e.g., radiowaves and microwaves) ${ }^{2}$.

He demonstrated radiowave-induced 1D depth-resolved imaging without intended lateral resolution but did not mention the possibility of optical excitation in his patent. In the 1990s, the laser-induced counterpart of 1D depth-resolved imaging was demonstrated $^{3-7}$. With inverse reconstruction or spherically focused ultrasonic detection, $2 \mathrm{D}^{8}$ and $3 \mathrm{D}^{9,10} \mathrm{PAT}$, with both lateral and axial resolutions, were finally developed. In the decade that followed, several milestones were reached in anatomical, functional, and molecular PAT ${ }^{11-15}$. The fast growth of the field was triggered particularly by the first functional photoacoustic computed tomography (PACT) ${ }^{11}$ and photoacoustic microscopy $(\mathrm{PAM})^{12}$ modalities.

Although turn-key commercial PAT systems are now available for preclinical applications, users can still benefit from understanding the principles and characteristics of PAT, especially when choosing or optimizing a PAT system for a specific application. In this Review, we introduce the basic principles of PAT and emphasize its functional and molecular imaging capabilities in the preclinical life sciences. We provide practical guidelines, with case studies, to scientists who want to choose or develop a PAT system that can best serve their research, and we summarize the most exciting biomedical applications. We also consider potential breakthroughs that may overcome current technical challenges of the PAT modality.

\section{Principles of PAT}

Although PAT has been tailored for diverse applications, its principles remain constant. The imaging process typically starts with a short laser pulse fired at biological tissue. As photons propagate into the tissue, some are absorbed by biomolecules (e.g., hemoglobin, DNA-RNA, lipids, water, melanin, and cytochromes). The various absorption mechanisms in PAT include (but are not limited to) electronic absorption, vibrational absorption ${ }^{16}$, stimulated Raman absorption ${ }^{17}$, and surface plasmon resonance absorption ${ }^{18}$. The absorbed optical energy is usually partially or completely converted into heat through nonradiative relaxation of excited molecules (Fig. 1a). The heat-induced pressure wave propagates in tissue as an ultrasound wave. The ultrasound wave is detected outside the tissue by an ultrasonic transducer or transducer array to form an image that maps the original optical energy deposition inside the tissue (Fig. 1b). PAT has a $100 \%$ relative sensitivity to small variations in optical absorption, which

\section{BOX 1 LIGHT SOURCES IN PAT}

PAM and PACT have different requirements for optimal pulsed lasers, mainly in their pulse repetition rate, pulse energy, pulse width, and wavelength tunability. In general, while a high pulse repetition rate is key to PAM, a high pulse energy output is important to PACT.

For raster scanning PAM (Fig. 2a-c), the laser pulse repetition rate should be more than $1 \mathrm{kHz}$ for rapid 3D imaging. For example, to raster scan $200 \times 200$ points within $0.1 \mathrm{~s}$, the pulse repetition rate must be at least $400 \mathrm{kHz}$. For PACT (Fig. $\mathbf{2 d - g}$ ), where a single pulse can generate a $2 \mathrm{D}$ or $3 \mathrm{D}$ image, a pulse repetition rate of tens of hertz is usually sufficient. With a relatively small optical excitation region, PAM usually employs less pulse energy ( $<1 \mu \mathrm{J}$ for $0 \mathrm{R}-\mathrm{PAM}$ and $<1 \mathrm{~mJ}$ for AR-PAM) than PACT $(>10 \mathrm{~mJ})$. For both PAM and PACT, the laser pulse width should be less than the acoustic transit time across the targeted axial resolution length (on the scale of nanoseconds). Finally, the laser wavelength should be tunable for spectroscopic functional and molecular PAT, where multiple PA images are acquired at different wavelengths. Q-switched $\mathrm{Nd}$ : YAG pumped OPO, Ti:Sapphire, and dye laser systems are most commonly used, as they provide sufficient pulse energies with nanosecond pulse widths at biologically relevant wavelengths. Unfortunately, the pulsed-laser sources are generally bulky and expensive. Recently, pulsed-laser diodes ${ }^{102}$ and lightemitting diodes ${ }^{103}$ have been reported as compact and less expensive alternatives; however, they have low pulse energies even when used in groups (e.g., stacking multiple laser diodes), resulting in low signal-to-noise ratio. Employing temporally coded excitation (e.g., Golay $\operatorname{codes}^{103}$ ) improves signal-to-noise ratio at the cost of imaging speed. 
Figure 2 | Representative implementations of PAT. (a) Transmission-mode OR-PAM system, where the ultrasonic transducer (UT) and the water-immersion focusing lens are on opposite sides of the object ${ }^{98}$. Note that the focusing lens has a numerical aperture (NA) of 1.2 and a working distance of only $\sim 200 \mu \mathrm{m}$. (b) Reflection-mode OR-PAM system with an optical-acoustic combiner that transmits light but reflects sound ${ }^{96}$. SOL, silicone oil layer sandwiched between two prisms. (c) AR-PAM system with a dark-field illumination ${ }^{12}$. The laser light is only weakly focused, with the UT in the dark cone. (d) PACT system with a ringshaped ultrasonic transducer array (UTA) ${ }^{99}$. The laser beam is expanded and homogenized by a diffuser to provide widefield illumination. (e) PACT system with a linear UTA ${ }^{100}$. The excitation light is delivered through a fusedend, bifurcated fiber bundle that flanks both sides of the UTA. (f) PACT system with a hemispherically shaped UTA ${ }^{29}$. The UTA is rotated around the object to be imaged to provide dense spatial sampling for 3D imaging.

(g) PACT system with a 2D Fabry-Perot interferometer as the acoustic sensor ${ }^{70}$. The PA

waves are recorded by raster scanning a probing laser beam over the surface of the interferometer. (h) Side-viewing intravascular PA catheter with an outer diameter of $1.25 \mathrm{~mm}$, including the protective sheath in which the catheter rotates ${ }^{23}$. Note that the acoustic-coupling medium (typically water or ultrasound gel) is not shown in the schematics.

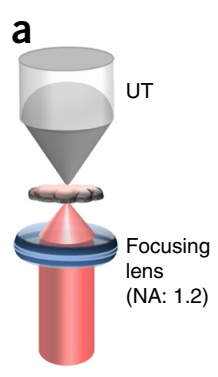

b
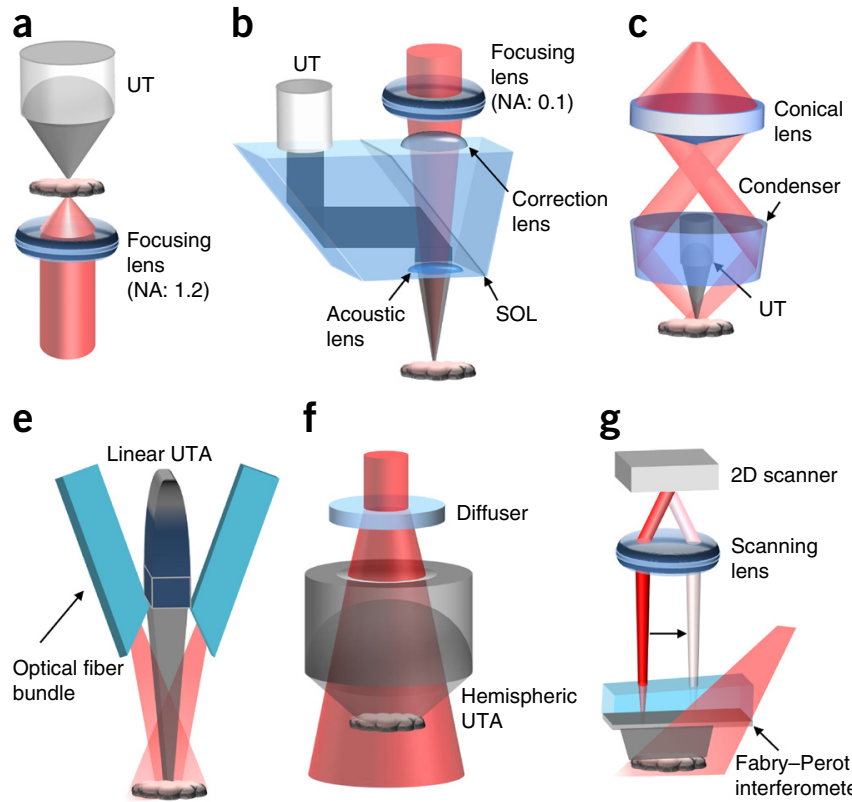$$
\text { g }
$$

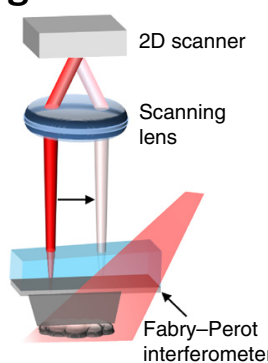

h
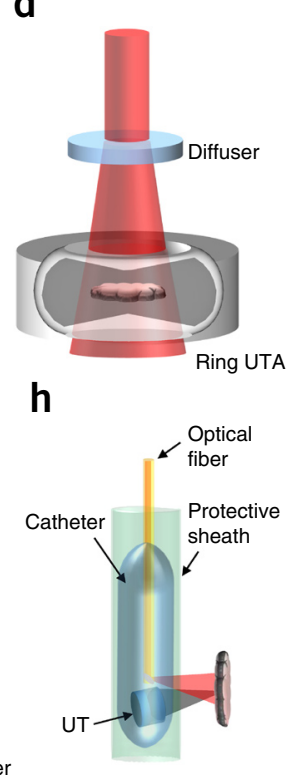

means that a given percentage change in the optical absorption coefficient yields the same percentage change in the PA signal amplitude. By contrast, back-scattering-based confocal microscopy has a relative sensitivity to optical absorption in blood of only $\sim 6 \%$ at $560 \mathrm{~nm}$ and $\sim 0.08 \%$ at $800 \mathrm{~nm}$. Because PAT does not rely on fluorescence, which usually has a quantum yield of less than $100 \%$, it can image nearly all molecules ${ }^{15,19,20}$.

Major PAT implementations. The major components of a typical PAT system include a short-pulsed laser (e.g., a nanosecond Q-switched Nd:YAG laser) for efficient wideband PA signal generation (Box 1); a wideband ultrasonic transducer or transducer array for signal detection; a data-acquisition system for signal amplification and digitization; and a computer for system synchronization, data collection, and image formation. The bandwidth of the transducer should match the bandwidth of the PA signals that originate from small optical absorbers at the desired depth and survive the frequency-dependent acoustic attenuation of tissue. Matched broadband detection optimizes signal-to-noise ratio and thus detection sensitivity and axial resolution.

PAT has been implemented with two major image-formation methods (Fig. 2). The first method, focused-scanning image formation, commonly used in PAM, is based on mechanical scanning of a focused excitation light beam and a focused singleelement ultrasonic transducer (Fig. 2a-c). The second method, used in PACT, is inverse reconstruction image formation, based on widefield light illumination and acoustic detection at multiple locations (Fig. 2d-g). PAT can also be implemented for endoscopy ${ }^{21-24}$. On account of the high scalability of PAT, the imaging performances of different implementations are highly overlapping, with convenient transitions among them. In addition, complementary original implementations can be hybridized in various forms. Furthermore, because it combines optical excitation with acoustic detection, PAT is compatible with complementary imaging modalities, especially optical imaging and ultrasound imaging ${ }^{25}$.

PAM can be further classified into optical-resolution PAM (ORPAM) (Fig. 2a,b), where the optical focus is much tighter than the acoustic focus, and acoustic-resolution PAM (AR-PAM) (Fig. 2c), where the acoustic focus is tighter than the diffused optical beam. In both cases, the axial resolution is determined acoustically. In OR-PAM, the optically determined lateral resolution is typically much finer than the axial resolution. The optical focusing can be either strong or weak, depending on the desired spatial resolution and penetration. Conversely, in AR-PAM, the axial resolution is typically finer than the lateral resolution.

PACT can be configured with a 1D ultrasonic transducer $\operatorname{array}^{26,27}$ (Fig. 2d,e), a 2D transducer array (Fig. 2f) ${ }^{28,29}$, or their scanning equivalents (Fig. 2g) $)^{11,30,31}$. For 1D-array-based PACT, the axial (or radial) and lateral (or tangential) resolutions in the imaging plane, derived from reconstruction, are typically finer than the elevational resolution, which is determined by cylindrical acoustic focusing. In the imaging plane, the axial (or radial) direction is along the axis that perpendicularly passes the transducer element surface through the center (i.e., along the acoustic axis), and the lateral (or tangential) direction is perpendicular to the axial (or radial) direction. The elevational direction is orthogonal to the imaging plane. For 2D-array-based PACT, the resolutions can be nearly isotropic at the center of the field of view. Compared with PAM, state-of-the-art PACT generally has higher imaging speeds and greater penetration, but lower spatial resolutions.

Image formation in PAT. PAT forms images by numerically reversing the received PA signals in time back to their original 
Figure 3 | Practical guide to mapping the desired imaging depth, speed, and contrast to the optimal PAT implementation. For a specific biological problem, the most suitable category of PAT implementations depends primarily on the desired imaging depth. A reference representing each PAT implementation is indicated in brackets.

positions in space using the known speed of sound. In PAM (Fig. 2a-c), each PA signal is received primarily from the volume laterally confined by the optical focus or acoustic focus, whichever is smaller; it is converted into a 1D image of the optical energy deposition along the acoustic axis by extracting its envelope-the amplitude modulation curve of the radio-frequency PA signal. In PACT (Fig. 2d-g), each ultrasonic transducer element receives $\mathrm{PA}$ signals over a large acceptance angle, and data from all transducer elements are used to reconstruct an image using the inverse spherical Radon transformation, which is similar to triangulation used in the Global Positioning System ${ }^{32,33}$.

Depending on the image-formation method, PAT may require mechanical or electronic scanning. For PAM, where a single laser pulse generates a $1 \mathrm{D}$ depthresolved image, $2 \mathrm{D}$ raster scanning or arbitrary trajectory scanning of the ultrasonic transducer is employed to obtain a $3 \mathrm{D}$ image. For PACT with a $1 \mathrm{D}$ transducer array, where a single laser pulse generates a $2 \mathrm{D}$ cross-sectional image, orthogonal scanning along the elevational direction or rotational scanning around the object is needed to obtain a 3D image. For PACT with a $2 \mathrm{D}$ transducer array, a single laser shot can theoretically generate a 3D image ${ }^{28}$; in practice, however, either electronic or mechanical scanning of the transducer array is needed to complete data acquisition.

\section{Matching PAT systems with applications}

A given study may be best matched with a particular PAT system. The three typical questions regarding a study's compatibility with a given PAT system concern (1) imaging depth, (2) imaging speed or temporal resolution, and (3) imaging contrast. To best address a specific problem, one may need to optimally trade off the performance parameters of PAT. As a guide, Figure 3 categorizes the major PAT implementations according to these three criteria-depth, speed, and contrast of interest.

Imaging depth. Photon propagation in soft tissue can be loosely classified into four regimes, which define the penetration limits of various high-resolution optical imaging modalities (Fig. 4a) ${ }^{34}$. The spatial resolution of PAT scales with the imaging depth over a wide range from the quasi-ballistic regime (typically $\leq 1 \mathrm{~mm}$ in tissue) to the diffusive regime (typically $\geq 10 \mathrm{~mm}$ in tissue), up to the dissipation limit $(\sim 10 \mathrm{~cm} \text { in tissue })^{35}$. Reducing the imaging depth of PAT improves its spatial resolution in approximate proportion. As a rule of thumb, the ratio of the imaging depth to the resolution is $\sim 200$, permitting PAT to provide high-resolution imaging across a wide range of imaging depths (Fig. 3).

Considering the prevalence of confocal microscopy and twophoton microscopy in the life sciences, we illustrate how these techniques compare with PAM and PACT in terms of imaging depth (Fig. 4b). The imaging depth of confocal microscopy is limited to $100-200 \mu \mathrm{m}$ in biological tissue because it relies on the miniscule number of ballistic photons that have survived the roundtrip optical attenuation. Two-photon microscopy improves the imaging depth by using longer excitation wavelengths, which reduces optical attenuation and broadens optical focusing. Nevertheless, the low two-photon excitation efficiency and the overwhelming surface signal still limit the penetration to $\sim 1.5 \mathrm{~mm}$. Compared with two-photon microscopy, OR-PAM can achieve a similar, if not greater, imaging depth for two reasons: (1) the signal amplitude depends linearly instead of quadratically on the excitation-light intensity, and the detection suffers negligible acoustic attenuation; and (2) time-resolved acoustic detection effectively suppresses the interference of the surface signal. Nevertheless, OR-PAM still relies on quasi-ballistic photons 
Figure 4 | Photon propagation regimes in soft tissue and the penetration limits of representative high-resolution optical imaging modalities. (a) Photon propagation regimes in soft tissue and association with the penetration limits of high-resolution optical imaging modalities ${ }^{34}$. The four regimes are divided at photon propagation depths of approximately $0.1 \mathrm{~mm}$ (aberration limit), $1 \mathrm{~mm}$ (diffusion limit), $10 \mathrm{~cm}$ (dissipation limit), and $1 \mathrm{~m}$ (absorption limit), with an optical absorption coefficient of $0.1 \mathrm{~cm}^{-1}$, optical scattering coefficient of $100 \mathrm{~cm}^{-1}$ and anisotropy of 0.9 . The classification holds in optical-scatteringdominant media. Note that the penetration limits shown here are order-of-magnitude approximations and the penetration of wavefront engineering is a theoretical projection. (b) Signal generation and detection in confocal microscopy (CFM), two-photon microscopy (TPM) and PAT, with different penetration limits in scattering tissue. The colors shown for the excitation light do not represent the true optical wavelengths.

to provide optically defined lateral resolution. If the spatial resolution is relaxed to tens of micrometers, which is achievable with high-frequency ultrasonic detection, AR-PAM can be used to break into the quasi-diffusive regime (e.g., a penetration of $\sim 5 \mathrm{~mm}$ at $50 \mathrm{MHz}$ ultrasonic detection $^{36}$ ), where most photons have experienced many scattering events. With acoustically defined lateral resolution, both ballistic and scattered photons that reach the target contribute to the PA signal. The penetration of AR-PAM is largely limited by high-frequency acoustic attenuation and can potentially be extended to at least $10 \mathrm{~mm}$ with proportionately relaxed resolutions ${ }^{37}$. If the desired spatial resolution is further relaxed to hundreds of micrometers, which is attainable with low-frequency ultrasonic detection (e.g., $5 \mathrm{MHz}$ ), PACT can be used to reach the diffusive regime with penetration demonstrated up to $70 \mathrm{~mm}$ in living tissue ${ }^{38}$. However, PACT is still limited by dissipation to less than $\sim 10 \mathrm{~cm}$ in soft tissue, where the optical fluence-the photon energy received per unit area $\left(\mathrm{J} / \mathrm{cm}^{2}\right)$-is substantially attenuated on account of both absorption and scattering. When the optical scattering effect is compensated for by using wavefront engineering technologies ${ }^{39}$, it may be possible to approach a sufficient penetration toward the absorption limit ( 1 m) for whole-body human imaging (Fig. 4a).

Spatial resolution. Once a desired imaging depth is determined, one can estimate the achievable spatial resolution (Fig. 5a). For imaging in the quasi-ballistic regime, OR-PAM is preferred, as it can resolve single cells or organelles (Fig. 5b,c). OR-PAM is useful for studying cancer angiogenesis, where neocapillaries are less than $10 \mu \mathrm{m}$ in diameter, and for single-cell flowmetry, where the functions of individual cells are of interest ${ }^{40}$. AR-PAM is preferred for imaging in the quasi-diffusive regime. An imaging depth of $\sim 5 \mathrm{~mm}$ and tissue-level ( $\sim 50-\mu \mathrm{m}$ lateral and $\sim 15-\mu \mathrm{m}$ axial) spatial

b
Optical propagation depth

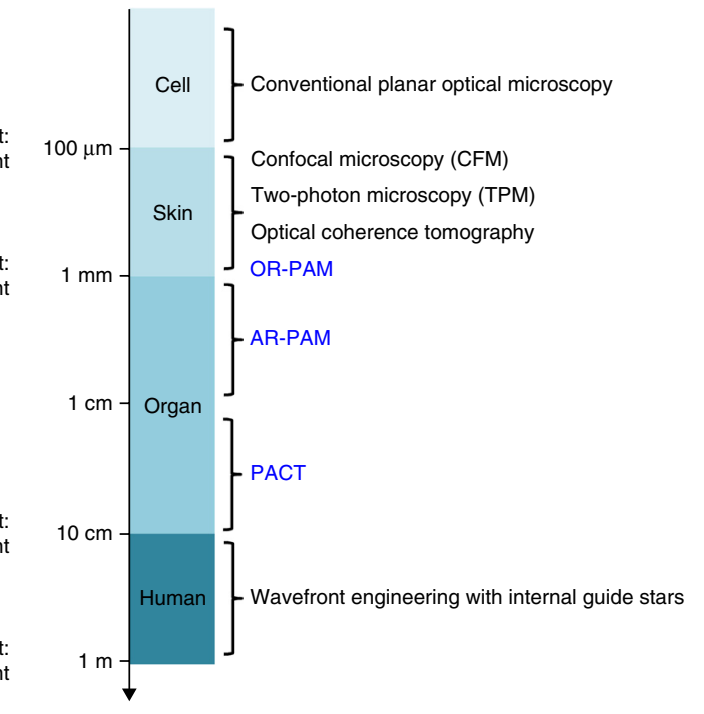

Absorption limit:

ring coefficient ing coefficient

issipation limit: ation coefficien

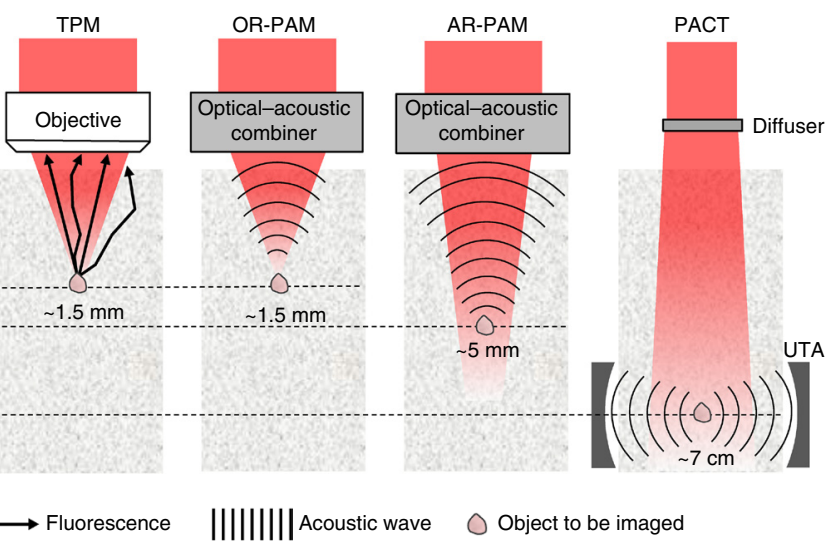

resolutions have been achieved (Fig. 5d) ${ }^{12}$. For imaging in the diffusive regime, reaching whole-body depths for small animals (Fig. 5e), PACT with spatial resolution of hundreds of micrometers is preferred (Fig. 5f $)^{31}$.

The spatial resolution of PAT can be tuned optically and acoustically (Fig. 5a). For OR-PAM, a shorter excitation wavelength and tighter optical focusing yield finer lateral resolution, and a wider ultrasonic detection bandwidth in megahertz provides better axial resolution. Nevertheless, it is worth noting that the axial resolution of OR-PAM with a tens-of-megahertz detection bandwidth is on the level of tens of micrometers. The recent development of OR-PAM with an ultrawide detection bandwidth (in gigahertz) has provided approximately isotropic resolutions ${ }^{41}$. However, the strong acoustic attenuation at ultrahigh ultrasound frequencies limits the applications to single-cell studies. For AR-PAM, a wider ultrasonic detection bandwidth similarly provides better axial resolution, while a higher central ultrasonic detection frequency benefits lateral resolution. Furthermore, a tighter acoustic focusing improves lateral resolution at the cost of focal zone, which is defined as the depth range within which the lateral resolution degrades up to $\sim 1.4 \times$. The spatial resolutions of PACT can be improved by increasing the central frequency and bandwidth of the ultrasonic transducer array. However, in all cases, spatial resolution is improved at the expense of penetration. 
Figure 5 | Multiscale PAT of single cells, whole-body small animals, and humans. (a) PAT implementations with approximate spatial resolutions and penetration limits suitable for structures ranging from organelles to whole-body small animals and humans. SR-PAM, super-resolution PAM. Note that the penetration limits and spatial resolutions shown here are order-of-magnitude approximations. (b) SR-PAM image of a single mitochondrion $^{42}$. Scale bar, $300 \mathrm{~nm}$. (c) OR-PAM image of individual red blood cells ${ }^{98}$. Scale bar, $7 \mu \mathrm{m}$. (d) In vivo AR-PAM image of subcutaneous vasculature of the palm of a human hand ${ }^{12}$. Scale bar, $1 \mathrm{~mm}$. (e) In vivo whole-body PACT image of a mouse, showing blood-rich internal organs ${ }^{37}$. Scale bar, $5 \mathrm{~mm}$. (f) In vivo PACT image of a human hand, showing its comprehensive vasculature. Image courtesy of Canon (Japan) (http:// www.canon.com/technology/future/index.html).

Super-resolution OR-PAM that beats the optical diffraction limit has recently been achieved. Various nonlinear mechanisms have been used to increase resolution, including optical absorption saturation (Fig. 5b $)^{42}$, photothermal nonlinearity ${ }^{43}$, two-photon absorption ${ }^{44}$, nanobubble cavitation ${ }^{45}$, thermal or Grueneisen relaxation $^{46}$, photothermal bleaching ${ }^{47}$, and reversible photoswitching ${ }^{48}$. Just as in confocal microscopy and twophoton microscopy, in addition to the enhancement in lateral resolution, nonlinear OR-PAM automatically enables optical sectioning, which provides at least ten-fold-finer axial resolution than the time-resolved acoustic sectioning in conventional linear OR-PAM.

Imaging speed. Biological functions occur on a wide variety of temporal scales. Various implementations of PAT offer a wide range of imaging speeds with associated tradeoffs (Fig. 3). Scientists should choose imaging systems with only the imaging speeds needed so that the other performance parameters can be optimized.

For OR-PAM (Fig. 2a,b), different scanning mechanisms can be employed according to the desired imaging speeds ${ }^{49}$. Unlike confocal or two-photon microscopy, PAM does not require depth scanning for $3 \mathrm{D}$ imaging on account of its time-resolved acoustic detection. When video-rate $3 \mathrm{D}$ imaging is needed, the excitation laser beam can be raster scanned within the acoustic focal spot ( $~ 50 \mu \mathrm{m}$ in diameter), although this technique confines the field of view. A $3 \mathrm{D}$ imaging rate of $\sim 30 \mathrm{~Hz}$, limited largely by the laser pulse repetition rate, has been used to image single red blood cells flowing in capillaries. Random-access scanning over only the features of interest while skipping the background, enabled by a digital micromirror array, can further improve the imaging speed ${ }^{50}$. Unfocused acoustic detection can enlarge the field of view-up to $\sim 6 \mathrm{~mm}$ in diameter as demonstrated thus far - at the expense of signal amplitude ${ }^{51}$. Recently, hybrid scanning using a water-immersible microelectromechanical system (MEMS) mirror that confocally steers both the excitation laser beam and the emitted acoustic beam has achieved a 3D imaging rate of $\sim 1 \mathrm{~Hz}$, with a moderate field of view of $\sim 3 \times 4 \mathrm{~mm}^{2}$ and uncompromised detection sensitivity 52,53 . If lower-dimensional imaging suffices, the imaging rate improves with each reduced dimension by approximately two orders of magnitude.

For AR-PAM (Fig. 2c), the imaging speed is mainly limited by the mechanical scanning speed and the pulse repetition rate of the higher-pulse-energy laser, the latter of which is limited by safety of laser exposure on the tissue. In AR-PAM, mechanical scanning
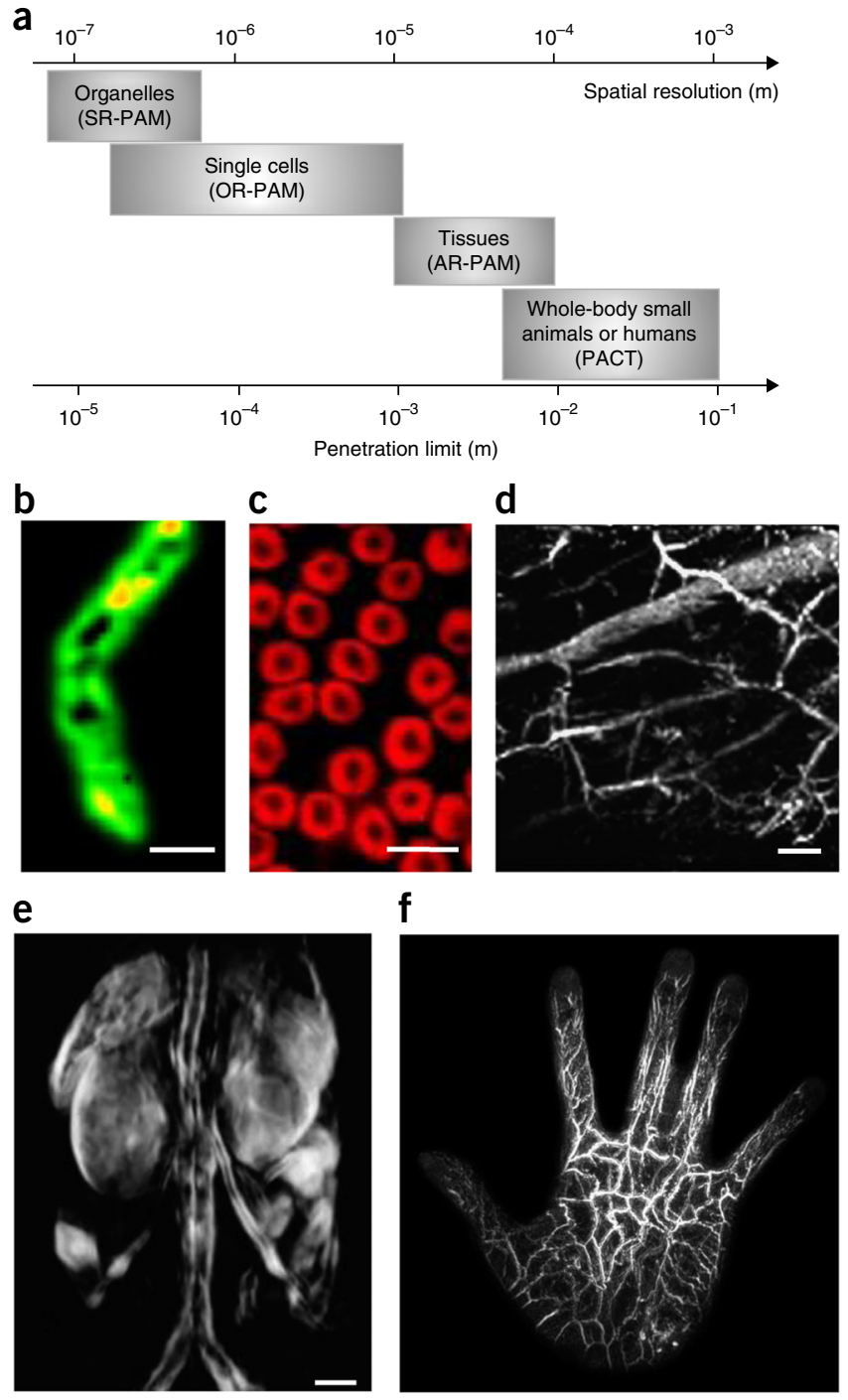

by a step motor or a voice-coil scanner is typically used with a scanning step size $\sim 10 \times$ that in OR-PAM. A 2 D imaging rate of $40 \mathrm{~Hz}$ has been achieved by AR-PAM over a scanning range of $\sim 9 \mathrm{~mm}$; this rate is sufficient to capture the oxygenation dynamics in a mouse heart within a heart beat ${ }^{36}$.

For PACT that provides a large field of view, the imaging speed is usually limited by the pulse repetition rate of the laser and the time required for multiplexed data acquisition. Using a $1 \mathrm{D}$ or $2 \mathrm{D}$ ultrasonic transducer array, a single laser shot can theoretically generate a $2 \mathrm{D}$ or $3 \mathrm{D}$ image over the entire field of view. However, multiplexed data acquisition, which is usually adopted to reduce costs, slows down data collection. Commercially available highpulse-energy lasers used in PACT typically operate at a repetition rate less than $50 \mathrm{~Hz}$. In addition, since repeated widefield illumination may cause tissue damage because of accumulated heating, the average fluence rate $\left(\mathrm{W} / \mathrm{cm}^{2}\right)$ in addition to the fluence $\left(\mathrm{J} / \mathrm{cm}^{2}\right)$ per pulse on the tissue surface should be controlled to prevent damage ${ }^{54}$. A recently developed PACT system with a hemispherical ultrasonic transducer array (256 elements) has achieved a $3 \mathrm{D}$ imaging rate of $\sim 50 \mathrm{~Hz}$ over a field of view of $15 \times$ $15 \times 7 \mathrm{~mm}^{3}$ (Fig. 2f), which is sufficient to image the beating heart of a mouse and the neuronal activities of a swimming zebra fish 
Figure 6 | In vivo PA molecular imaging. (a) PA images acquired at multiple wavelengths are combined with spectral unmixing algorithms to separate different types of optical absorbers ${ }^{101}$. This example shows an NIR organic dye (shown in green) taken up by the kidneys in a mouse model, with the strong background signals from oxyhemoglobin $\left(\mathrm{HbO}_{2}\right.$, shown in red) and deoxyhemoglobin (HbR, shown in blue). (b) Detection sensitivity of molecular probes. The detection sensitivities shown here are derived from reported results of representative contrast agents by adjusting them for both ANSI-limited light fluences and 3-mm depth. (c) PACT image of a kidney tumor expressing reversibly switchable nonfluorescent bacterial phytochrome BphP1 (ref. 48). The tumor is shown in color, and the background blood-rich organs are shown in gray. Scale bar, $1 \mathrm{~mm}$. a
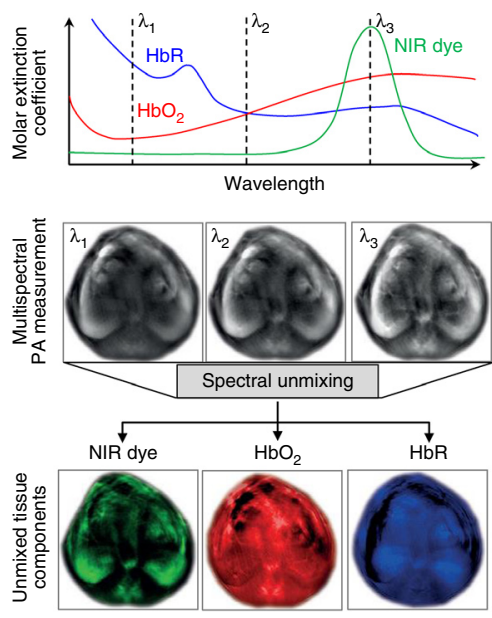

b

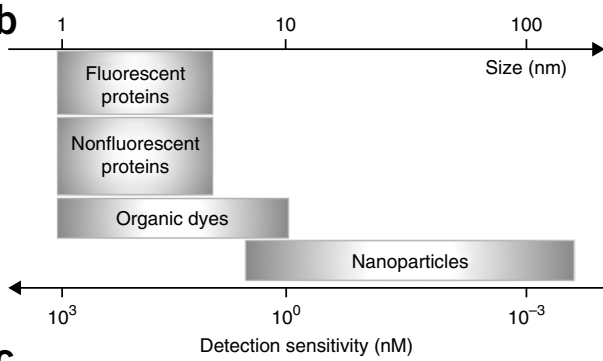

C

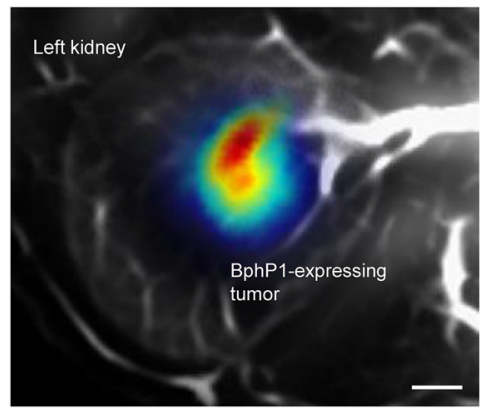

in real time ${ }^{28}$. However, the sparse spatial sampling due to the limited number of transducer elements induces imaging artifacts outside the central field of view. For PACT systems based on a Fabry-Pérot interferometer (Fig. 2g), the spatial resolutions can be well maintained with dense spatial sampling over the field of view, but the imaging speed is limited by the point-by-point scanning of the probing beam ${ }^{30}$. Widefield detection of the interference pattern using time-gated light illumination and a high-speed CCD camera can potentially speed up imaging ${ }^{55,56}$.

Imaging contrast and sensitivity. To study a given biological phenomenon, scientists also need to identify an optimal imaging contrast that can report the phenomenon with high sensitivity or specificity or both. Because PAT does not rely on fluorescence, any molecule can theoretically be imaged as PA contrast as long as nonradiative relaxation occurs. Both endogenous and exogenous contrast agents have been extensively explored by PAT ${ }^{57,58}$.

Endogenous contrast agents have several major advantages: (1) nontoxicity, (2) nonperturbation to biological processes, (3) abundance, and (4) freedom from regulatory approval. The most commonly imaged endogenous contrast agents in PAT include DNA-RNA ${ }^{59}$, hemoglobin ${ }^{31}$, melanin ${ }^{40}$, lipids $^{36}$, and water ${ }^{60}$ (Fig. 3). Among them, hemoglobin is most commonly used for label-free hemodynamic imaging, providing anatomic, functional, and metabolic information about blood-vessel diameter, total hemoglobin concentration, blood oxygenation, blood flow, and oxygen metabolism.

Exogenous contrast agents have two advantages over endogenous ones: (1) optimization for greater detection sensitivity and (2) conjugation with targeting molecules (e.g., antibodies) to selectively bind to receptors for molecular imaging. A wide range of exogenous contrast agents-including organic dyes $^{61}$, fluorescent proteins ${ }^{62}$, nonfluorescent proteins ${ }^{48}$, and

Table 1 | Major components and imaging parameters of subwavelength OR-PAM (SW-OR-PAM) and whole-body PACT (WB-PACT)

\begin{tabular}{llll}
\hline PAT & & SW-OR-PAM ${ }^{\mathbf{2}}$ & \multicolumn{1}{c}{ WB-PACT $^{\mathbf{3 1}}$} \\
\hline Optical excitation & Light source & $\mathrm{Nd}: \mathrm{YVO}_{4}$ laser & Alexandrite laser \\
& Pulse energy & $10 \mathrm{~nJ}$ & $100 \mathrm{~mJ}$ \\
& Pulse width & $1.5 \mathrm{~ns}$ & $75 \mathrm{~ns}$ \\
& Wavelength & $532 \mathrm{~nm}$ & $755 \mathrm{~nm}$ \\
& Pulse repetition rate & $16 \mathrm{kHz}$ & $10 \mathrm{~Hz}$ \\
& Beam shape & Tightly focused illumination $(\sim 220 \mathrm{~nm}$ in diameter $)$ & Widefield illumination $(\sim 8 \mathrm{~cm}$ in diameter $)$ \\
\hline Ultrasonic detection & Transducer & Single-element spherically focused transducer & Concave arc-shaped array with 64 elements \\
& Central frequency & $40 \mathrm{MHz}$ & $3.1 \mathrm{MHz}$ \\
& Bandwidth & $40 \mathrm{MHz}$ & $2.4 \mathrm{MHz}$ \\
\hline Scanning mechanism & & Raster scanning of the object to be imaged & Rotational scanning of the object to be imaged \\
\hline Data acquisition & Signal amplification & $48 \mathrm{~dB}$ & $70 \mathrm{~dB}$ \\
& Channel count & Single channel & $64 \mathrm{channels}$ \\
& Sampling rate & $1 \mathrm{GHz}$ & $40 \mathrm{MHz}$ \\
\hline Image formation & & Direct envelope extraction & Back-projection reconstruction \\
\hline Spatial resolution & Lateral & $0.22 \mu \mathrm{m}$ & $0.5 \mathrm{~mm}$ \\
& Axial & $30 \mu \mathrm{m}$ & $0.5 \mathrm{~mm}$ \\
\hline Imaging time (Field of view) & & $\sim 1 \mathrm{~min}\left(100 \times 100 \times 100 \mu \mathrm{m}^{3}\right)$ & $\sim 8 \mathrm{~min}\left(4 \times 4 \times 6 \mathrm{~cm}^{3}\right)$ \\
\hline Penetration limit & & $\sim 0.1 \mathrm{~mm}$ & $\sim 30 \mathrm{~mm}$ \\
\hline
\end{tabular}


Figure 7 | Representative in vivo PAT applications in life sciences. (a) Whole-cortex OR-PAM image of the oxygen saturation of hemoglobin in a mouse brain ${ }^{53}$. The arteries (shown in red) and veins (shown in blue/green) are clearly differentiated by their oxygenation levels. Blue indicates low oxygenation in some vessels. Scale bar, $1 \mathrm{~mm}$. (b) Sequential label-free OR-PAM images of oxygen releasing in single red blood cells (RBCs) flowing in a capillary in a mouse brain ${ }^{86}$. Scale bar, $10 \mu \mathrm{m}$. Blood flows from left to right. The dashed arrow follows the trajectory of a single flowing RBC. (c) PACT images of tyrosinase-expressing $\mathrm{K} 562$ tumor (shown in yellow) after subcutaneous injection into the flank of a nude mouse ${ }^{70}$. The surrounding blood vessels are shown in gray. Top, $x-y$ projection image; bottom, $y-z$ projection image. Scale bar, $1 \mathrm{~mm}$.
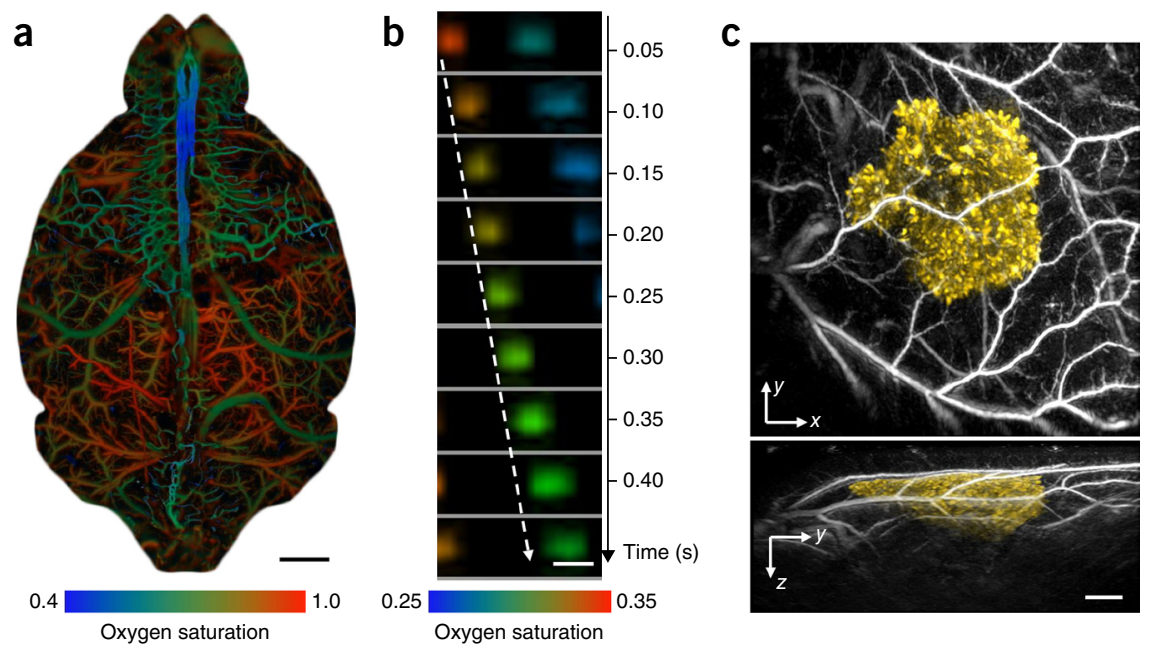

nanoparticles ${ }^{63}$ - have been investigated for high-sensitivity PA molecular imaging (Fig. 3).

Quantitative spectroscopic PA imaging, where the tissue is sequentially illuminated at multiple wavelengths, has been commonly used to separate signals from multiple types of optical absorbers (Fig. 6a) ${ }^{64}$. However, quantifying the absorber concentrations generally requires knowledge of the local optical fluence at different wavelengths, which is often difficult to estimate, especially in deep tissue, on account of the strong but usually unknown wavelength-dependent light attenuation. Other factors, including PA-signal-generation efficiency, nonlinear optical absorption, and nonuniform light illumination and acoustic attenuation, also complicate the quantification. Recently, statistical unmixing methods using adaptive matched filters have demonstrated better performance than the conventional least-squares-based methods, showing more robustness to the uncertainties of the background signals ${ }^{65}$. However, these methods are more suitable for identifying sparsely distributed absorbers than for quantifying their concentrations. For more detailed discussion, readers are referred to comprehensive reviews of inverse methods for quantitative spectroscopic PA imaging ${ }^{64}$.

To achieve high detection sensitivity at depths, contrast agents with large optical absorption cross-sections in the red or nearinfrared (NIR) spectral region are usually preferred (Fig. 6b), as is detailed in the companion review ${ }^{58}$. Because of their large particle sizes and/or the surface plasmon resonance effect, nanoparticles generally provide high sensitivity and thus have been widely studied for $\mathrm{PAT}^{14}$, although they usually have long circulating times in the bloodstream and poor clearance from the body. Organic dyes are also widely used as PA contrast agents on account of their relatively short circulating times and fast clearance from the body ${ }^{66}$. Genetically encodable proteins (fluorescent or not) have become increasingly popular in PA molecular imaging owing to their high selectivity and ease of exogenous delivery ${ }^{15,20,62,67-71}$. Novel methods using activatable nanoparticles or photoswitchable proteins have been reported (Fig. 6c) $)^{48,72}$, and these methods can effectively enhance detection sensitivity.

To further improve PA detection sensitivity, which is often quantified as the noise-equivalent detectable concentration of a contrast agent, one can maximize the target signal amplitude, suppress the background signal amplitude, optimize the electronics, and reduce the noise amplitude. Increasing the optical fluence and tuning the laser wavelength toward the absorption peak, in addition to choosing contrast agents with large optical absorption cross-sections, increases the target signal amplitude, as long as the temperature rise per laser pulse is within the safety range. The American National Standards Institute (ANSI) has detailed standards for laser exposure to the skin and eye ${ }^{54}$. Within the ANSI limit, PAT is safe for in vivo imaging because of the adequate conversion efficiency from temperature rise to acoustic pressure (i.e., a 1-mK temperature rise leads to a detectable 800 -Pa pressure rise). Also, matching the ultrasonic transducer with the detectable PA signal spectrum, which is primarily limited by the depth of the target, maximizes the detection efficiency. While the detection sensitivity of a conventional piezoelectric ultrasonic transducer degrades with decreasing element size, the sensitivity of optical sensors of acoustic waves, which is typically based on interferometry, does not. Therefore, optical detection holds great promise for high-sensitivity PAE, where the ultrasonic transducer must be miniaturized. The electronics of PAT systems can be optimized by matching the impedances of the ultrasonic transducers and the amplifiers, in addition to picking low-noise amplifiers. The noise level can be reduced by shortening the cables, grounding the amplifiers, and shielding external interferences.

\section{Selected applications}

PAT has found broad applications in the life sciences, especially in functional brain mapping, cancer diagnosis, tissue engineering, developmental biology, and cell biology, as comprehensively reviewed elsewhere ${ }^{19}$. Here, we sample only several representative application areas where PAT has played increasingly important roles. We also highlight two case studies in Table 1.

PAT has enabled functional and metabolic brain imaging at both microscopic and macroscopic scales, providing valuable information about brain vasculature ${ }^{73}$, oxygenation (Fig. 7a $)^{27}$, metabolism of oxygen and glucose ${ }^{74}$, resting-state connectivity ${ }^{75}$, and brain responses to various physiological and pathological challenges ${ }^{76-78}$. PAT can provide neuronal imaging using either endogenous contrast from lipids or exogenous contrast from dyes $^{79}$. In addition, PAT has been increasingly used to study smallanimal models of brain diseases, including strokes ${ }^{80}$, epilepsy ${ }^{81}$, 


\section{BOX 2 ENGINEERING CHALLENGES IN PAT IMPLEMENTATIONS}

As discussed above, when implementing a PAT system for a particular life sciences study, the major components, such as the light source, ultrasonic detection system, and scanning system, should be chosen according to the required imaging performance. Several typical engineering challenges in PAT implementations deserve attention. For OR-PAM, the major engineering challenge is the confocal alignment of the focused optical excitation and acoustic detection. Different optical-acoustic beam combiners have been developed to achieve confocal alignment with a low numerical aperture (NA) optical focusing lens ${ }^{49}$. Dark-field optical excitation and dark-field acoustic detection have also been used for confocal alignment ${ }^{59}$. However, this challenge remains for reflection-mode OR-PAM with a high-NA (>0.6) optical focusing lens, which has a very limited working distance ( $<1 \mathrm{~mm})$. A potential solution is to use a thin ultrasonic detector that is optically transparent, such as a microring resonator ${ }^{104}$. For AR-PAM, the major challenge is achieving efficient light delivery to the deep acoustic focus without causing thermal damage to the skin surface. Weakly focused dark-field optical excitation the best solution so far, although the optimal excitation pattern is largely determined by the targeted imaging depth ${ }^{105}$. For PACT, the major challenge is high-speed data acquisition with a multielement ultrasonic transducer array. While multichannel data acquisition systems ( $\geq 128$ channels) are commercially available, they are expensive. A multichannel acoustic waveguide with a single-channel signal detection system might reduce the complexity and cost of PACT ${ }^{106}$. and edemas ${ }^{60}$. Recently, lightweight head-mounted PACT systems have been developed, an important step toward functional PA imaging of brains in free-moving animals ${ }^{82}$.

High-resolution imaging of single cells is crucial in cell biology. OR-PAM, with submicrometer to micrometer resolution, is well suited for imaging single-cell morphology and function ${ }^{83}$. For cell morphology, OR-PAM can perform in vivo histologic imaging without staining by imaging DNA and RNA within the cell nuclei and cytochromes within the cytoplasm. Using ultrasonic transducers with extremely high frequencies $(>1 \mathrm{GHz})$, OR-PAM has revealed morphological abnormalities of diseased red blood cells and can differentiate white blood cell types according to the frequency features of their PA signals ${ }^{41,84,85}$. For cell function, PA oxymetry is capable of label-free imaging of oxygen unloading in single red blood cells in vivo (Fig. $7 \mathbf{b})^{86}$. PA flowmetry can detect circulating tumor cells in the bloodstream with high sensitivity and high throughput using endogenous contrast (e.g., melanin ${ }^{40,87}$ ) or targeted exogenous contrast (e.g., gold nanoparticles targeting breast cancer cells ${ }^{18}$ ).

PAT has been widely used for cancer studies ${ }^{88}$, including fundamental research in carcinogenesis ${ }^{89}$, cancer detection and staging ${ }^{63}$, and navigation and evaluation in cancer treatment ${ }^{90}$. In particular, PAT is an effective tool for label-free imaging of melanoma with melanin as the intrinsic contrast agent because melanoma cells have orders of magnitude stronger optical absorption in the red and NIR spectral regions than do red blood cells. OR-PAM can resolve single melanosomes in melanoma cells ${ }^{42}$, AR-PAM can monitor melanoma development in deep smallanimal brains ${ }^{91}$, and PACT has successfully imaged transgenic cancer cells expressing high-level melanin with a tyrosinase-based genetic reporter (Fig. 7c) ${ }^{70}$. For cancers that are devoid of highcontrast intrinsic pigments, targeted nanoparticles or organic dyes can provide exogenous contrast by selectively binding to the surface receptors of cancer cells $13,18,88,92-95$. In addition to the tumor itself, microenvironmental cancer hallmarks can be imaged. Both high-resolution PAM and deep-penetration PACT have provided label-free imaging of tumor angiogenesis and tumor hypoxia, two major cancer hallmarks ${ }^{13,96,97}$. The abnormal metabolisms of oxygen and glucose in cancers, also major cancer hallmarks, have been investigated by using PAT with endogenous and exogenous contrasts, respectively ${ }^{74,96}$.

\section{Outlook}

The strong momentum of PAT development is fueled by its increasingly popular applications in the life sciences and its commercialization for preclinical and clinical research. Several typical engineering challenges of PAT implementations and their solutions are summarized in Box 2.

To tap the full potential of PAT, we face technological challenges, but none are beyond our reach. (1) A single PAT system that fully integrates its multiscale-imaging capability with automatic image coregistration would be invaluable. A major challenge is the seamless combination of optical illumination and acoustic detection required for different imaging depths of interest. An optically transparent ultrasonic transducer or transducer array may help simplify the system configuration. (2) Miniaturized PAT systems are of great interest for imaging freely moving animals, especially for brain studies. The relatively low sensitivity of a miniaturized ultrasonic transducer and the limited options for fast acoustic scanning mechanisms are currently the major hurdles. High-speed wide-field optical detection of acoustic pressure might enable a solution. (3) Super-resolution PA methods with resolution finer than $50 \mathrm{~nm}$ are still needed to study subcellular structures such as dendritic spines. Such methods can be used for super-resolution imaging of both fluorescent and nonfluorescent molecules. The signal-to-noise ratio of the existing nonlinear PA methods is still insufficient to provide such high resolution at high speed. Donutshaped illumination-based methods, similar to stimulated emission depletion (STED) microscopy, could potentially be adapted to improve the resolution. (4) Single-molecule detection by PAT is highly desirable for molecular cell biology. Novel ultrasonic detectors with high piezoelectric efficiency and low acoustic impedance need to be developed. (5) Absolute quantitative PA measurement of oxygenation and temperature in deep tissue remains a challenge, mainly because of the unknown local optical fluence, along with other secondary factors such as tissue-dependent PA generation efficiency. New imaging methods and mathematical models are needed to better map the optical properties of the tissue. 
(6) Finally, for PAT of neuronal activities in the deep brain, novel genetically encodable indicators of action potentials or surrogates (e.g., voltage- or calcium-sensitive proteins) with large absorption cross-sections in the NIR spectral region must be found in nature or engineered from existing proteins. Along this line, nonfluorescent NIR phytochromes (e.g., RpBphP) could potentially be engineered as PA imaging contrasts to report action potentials. The strong acoustic attenuation and aberration by the skull (especially the side skull) is another challenge for single-neuron PAT of the deep brain. A potential solution for this challenge is to integrate PAT with X-ray CT, which provides data for accurate 3D modeling of the skull for acoustic correction.

PAT provides a comprehensive toolbox for the life sciences, complementing other imaging methods in its contrast mechanism, spatial-temporal resolution, and penetration. We expect PAT to find more high-impact applications in biomedical research.

\section{ACKNOWLEDGMENTS}

We thank J. Ballard for reading the manuscript and R. Zhang for preparing the figures. This work was sponsored by the US National Institutes of Health grants DP1 EB016986 (NIH Director's Pioneer Award), R01 CA186567 (NIH Director's Transformative Research Award), and U01 NS090579 (BRAIN Initiative), all to L.V.W.

\section{COMPETING FINANCIAL INTERESTS}

The authors declare competing financial interests: details are available in the online version of the paper.

Reprints and permissions information is available online at http://www.nature. com/reprints/index.html.

1. Bell, A.G. Upon the production and reproduction of sound by light. Am. J. Sci. 20, 305-324 (1880).

2. Bowen, T. Radiation-induced thermoacoustic imaging. US Patent 4,385,634 (1983).

3. Oraevsky, A.A., Jacques, S.L. \& Tittel, F.K. Determination of tissue optical properties by piezoelectric detection of laser-induced stress waves. In Laser-Tissue Interaction IV (Proceedings of the SPIE, vol. 1882) (eds. Jacques, S.L. \& Katzir, A.) 86-101 (SPIE, 1993).

4. Kruger, R.A. \& Liu, P.Y. Photoacoustic ultrasound: pulse production and detection of 0.5\% Liposyn. Med. Phys. 21, 1179-1184 (1994).

5. Kruger, R.A. Photoacoustic ultrasound. Med. Phys. 21, 127-131 (1994).

6. Oraevsky, A.A., Esenaliev, R.O., Jacques, S.L., Thomsen, S.L. \& Tittel, F.K. Lateral and $z$-axial resolution in laser optoacoustic imaging with ultrasonic transducers. In Optical Tomography, Photon Migration, and Spectroscopy of Tissue and Model Media: Theory, Human Studies, and Instrumentation (Proceedings of the SPIE, vol. 2389) (eds. Chance, B. \& Alfano, R.R.) 198-208 (SPIE, 1995).

7. Oraevsky, A.A., Esenaliev, R.O., Jacques, S.L. \& Tittel, F.K. Laser optoacoustic tomography for medical diagnostics: principles. In Biomedical Sensing, Imaging, and Tracking Technologies I (Proceedings of the SPIE, vol. 2676) (eds. Lieberman R.A., Podbielska, H. \& Vo-Dinh, T.) 84-90 (SPIE, 1996).

8. Kruger, R.A., Liu, P.Y., Fang, Y.R. \& Appledorn, C.R. Photoacoustic ultrasound (Paus)-reconstruction tomography. Med. Phys. 22, 1605-1609 (1995).

9. Hoelen, C.G.A., de Mul, F.F.M., Pongers, R. \& Dekker, A. Threedimensional photoacoustic imaging of blood vessels in tissue. Opt. Lett. 23, 648-650 (1998).

10. Wang, L.V., Zhao, X., Sun, H. \& Ku, G. Microwave-induced acoustic imaging of biological tissues. Rev. Sci. Instrum. 70, 3744-3748 (1999).

11. Wang, X.D. et al. Noninvasive laser-induced photoacoustic tomography for structural and functional in vivo imaging of the brain. Nat. Biotechnol. 21, 803-806 (2003).

12. Zhang, H.F., Maslov, K., Stoica, G. \& Wang, L.V. Functional photoacoustic microscopy for high-resolution and noninvasive in vivo imaging. Nat. Biotechnol. 24, 848-851 (2006).

13. Li, M.-L. et al. Simultaneous molecular and hypoxia imaging of brain tumors in vivo using spectroscopic photoacoustic tomography. Proc. IEEE 96, 481-489 (2008).

14. De la Zerda, A. et al. Carbon nanotubes as photoacoustic molecular imaging agents in living mice. Nat. Nanotechnol. 3, 557-562 (2008).
15. Razansky, D. et al. Multispectral opto-acoustic tomography of deep-seated fluorescent proteins in vivo. Nat. Photonics 3, 412-417 (2009).

16. Wang, H.W. et al. Label-free bond-selective imaging by listening to vibrationally excited molecules. Phys. Rev. Lett. 106, 238106 (2011).

17. Yakovlev, V.V. et al. Stimulated Raman photoacoustic imaging. Proc. Natl. Acad. Sci. USA 107, 20335-20339 (2010).

18. de la Zerda, A., Kim, J.W., Galanzha, E.I., Gambhir, S.S. \& Zharov, V.P. Advanced contrast nanoagents for photoacoustic molecular imaging, cytometry, blood test and photothermal theranostics. Contrast Media Mol. Imaging 6, 346-369 (2011).

19. Zackrisson, S., van de Ven, S.M.W.Y. \& Gambhir, S.S. Light in and sound out: emerging translational strategies for photoacoustic imaging. Cancer Res. 74, 979-1004 (2014).

20. Burgholzer, P., Grün, H. \& Sonnleitner, A. Photoacoustic tomography: sounding out fluorescent proteins. Nat. Photonics 3, 378-379 (2009).

21. Yang, J.M. et al. Simultaneous functional photoacoustic and ultrasonic endoscopy of internal organs in vivo. Nat. Med. 18, 1297-1302 (2012).

22. Bai, X.S. et al. Intravascular optical-resolution photoacoustic tomography with a $1.1 \mathrm{~mm}$ diameter catheter. PLOS ONE 9, e92463 (2014).

23. Jansen, K., van der Steen, A.F.W., van Beusekom, H.M.M., Oosterhuis, J.W. \& van Soest, G. Intravascular photoacoustic imaging of human coronary atherosclerosis. Opt. Lett. 36, 597-599 (2011).

24. Zhang, E.Z. \& Beard, P.C. A miniature all-optical photoacoustic imaging probe. In Photons Plus Ultrasound: Imaging and Sensing 2011 (Proceedings of the SPIE, vol. 7899) (eds. Oraevsky, A.A. \& Wang, L.V.) 78991F (SPIE, 2011).

25. Kim, J., Lee, D., Jung, U. \& Kim, C. Photoacoustic imaging platforms for multimodal imaging. Ultrasonography 34, 88-97 (2015).

26. Vionnet, L. et al. 24-MHz scanner for optoacousticimaging of skin and burn. IEEE Trans. Med. Imaging 33, 535-545 (2014).

27. Burton, N.C. et al. Multispectral opto-acoustic tomography (MSOT) of the brain and glioblastoma characterization. Neuroimage 65, 522-528 (2013).

28. Deán-Ben, X.L. \& Razansky, D. Adding fifth dimension to optoacoustic imaging: volumetric time-resolved spectrally enriched tomography. LightSci. Appl. 3, e137 (2014).

29. Kruger, R.A., Lam, R.B., Reinecke, D.R., Del Rio, S.P. \& Doyle, R.P. Photoacoustic angiography of the breast. Med. Phys. 37, 6096-6100 (2010).

30. Zhang, E., Laufer, J. \& Beard, P. Backward-mode multiwavelength photoacoustic scanner using a planar Fabry-Perot polymer film ultrasound sensor for high-resolution three-dimensional imaging of biological tissues. Appl. Opt. 47, 561-577 (2008).

31. Brecht, H.P. et al. Whole-body three-dimensional optoacoustic tomography system for small animals. J. Biomed. Opt. 14, 064007 (2009).

32. Xu, M.H. \& Wang, L.V. Time-domain reconstruction for thermoacoustic tomography in a spherical geometry. IEEE Trans. Med. Imaging 21, 814-822 (2002).

33. Treeby, B.E. \& Cox, B.T. k-Wave: MATLAB toolbox for the simulation and reconstruction of photoacoustic wave fields. J. Biomed. Opt. 15, 021314 (2010).

34. Yao, J., Xia, J. \& Wang, L.V. Multiscale functional and molecular photoacoustic tomography. Ultrason. Imaging 38, 44-62 (2016).

35. Wang, L.V. \& Hu, S. Photoacoustic tomography: in vivo imaging from organelles to organs. Science 335, 1458-1462 (2012).

36. Wang, L.D., Maslov, K., Xing, W.X., Garcia-Uribe, A. \& Wang, L.V. Video-rate functional photoacoustic microscopy at depths. J. Biomed. Opt. 17, 106007 (2012).

37. Jeon, M., Kim, J. \& Kim, C. Multiplane spectroscopic whole-body photoacoustic imaging of small animals in vivo. Med. Biol. Eng. Comput. 54, 283-294 (2014)

38. Mitcham, T., Dextraze, K., Taghavi, H., Melancon, M. \& Bouchard, R. Photoacoustic imaging driven by an interstitial irradiation source. Photoacoustics 3, 45-54 (2015).

39. Horstmeyer, R., Ruan, H.W. \& Yang, C.H. Guidestar-assisted wavefrontshaping methods for focusing light into biological tissue. Nat. Photonics 9, 563-571 (2015).

40. Galanzha, E.I., Shashkov, E.V., Spring, P.M., Suen, J.Y. \& Zharov, V.P. In vivo, noninvasive, label-free detection and eradication of circulating metastatic melanoma cells using two-color photoacoustic flow cytometry with a diode laser. Cancer Res. 69, 7926-7934 (2009).

41. Strohm, E.M., Berndl, E.S. \& Kolios, M.C. High frequency label-free photoacoustic microscopy of single cells. Photoacoustics 1, 49-53 (2013).

42. Danielli, A. et al. Label-free photoacoustic nanoscopy. J. Biomed. Opt. 19, 086006 (2014). 
43. Goy, A.S. \& Fleischer, J.W. Resolution enhancement in nonlinear photoacoustic imaging. Appl. Phys. Lett. 107, 211102 (2015).

44. Lee, S.-Y. et al. In vivo sub-femtoliter resolution photoacoustic microscopy with higher frame rates. Sci. Rep. 5, 15421 (2015).

45. Nedosekin, D.A., Galanzha, E.I., Dervishi, E., Biris, A.S. \& Zharov, V.P. Super-resolution nonlinear photothermal microscopy. Small 10, 135-142 (2014).

46. Wang, L., Zhang, C. \& Wang, L.V. Grueneisen relaxation photoacoustic microscopy. Phys. Rev. Lett. 113, 174301 (2014).

47. Yao, J.J., Wang, L.D., Li, C.Y., Zhang, C. \& Wang, L.V. Photoimprint photoacoustic microscopy for three-dimensional label-free subdiffraction imaging. Phys. Rev. Lett. 112, 014302 (2014).

48. Yao, J. et al. Multiscale photoacoustic tomography using reversibly switchable bacterial phytochrome as a near-infrared photochromic probe. Nat. Methods 13, 67-73 (2016).

49. Yao, J.J. \& Wang, L.V. Photoacoustic microscopy. Laser Photonics Rev 7 758-778 (2013).

50. Liang, J.Y. et al. Random-access optical-resolution photoacoustic microscopy using a digital micromirror device. Opt. Lett. 38, 2683-2686 (2013).

51. Song, W. et al. Integrating photoacoustic ophthalmoscopy with scanning laser ophthalmoscopy, optical coherence tomography, and fluorescein angiography for a multimodal retinal imaging platform. J. Biomed. Opt. 17, 061206 (2012).

52. Kim, J.Y., Lee, C., Park, K., Lim, G. \& Kim, C. Fast optical-resolution photoacoustic microscopy using a 2-axis water-proofing MEMS scanner. Sci. Rep. 5, 7932 (2015).

53. Yao, J. et al. High-speed label-free functional photoacoustic microscopy of mouse brain in action. Nat. Methods 12, 407-410 (2015).

54. American National Standards Institute. American National Standard for SafeUse of Lasers, ANSI Z136.1 (American National Standard Institute, 2007).

55. Nuster, R., Zangerl, G., Haltmeier, M. \& Paltauf, G. Full field detection in photoacoustic tomography. Opt. Express 18, 6288-6299 (2010).

56. Lamont, M. \& Beard, P.C. 2D imaging of ultrasound fields using CCD array to map output of Fabry-Perot polymer film sensor. Electron. Lett. 42, 187-189 (2006)

57. Luke, G.P., Yeager, D. \& Emelianov, S.Y. Biomedical applications of photoacoustic imaging with exogenous contrast agents. Ann. Biomed. Eng. 40, 422-437 (2012).

58. Weber, J., Beard, P.C. \& Bohndiek, S.E. Contrast agents for molecular photoacoustic imaging. Nat. Methods 13, 639-650 (2016).

59. Yao, D.K., Chen, R.M., Maslov, K., Zhou, Q.F. \& Wang, L.V. Optimal ultraviolet wavelength for in vivo photoacoustic imaging of cell nuclei. J. Biomed. Opt. 17, 056004 (2012).

60. Xu, Z., Zhu, Q.I. \& Wang, L.V. In vivo photoacoustic tomography of mouse cerebral edema induced by cold injury. J. Biomed. Opt. 16, 066020 (2011).

61. Akers, W.J. et al. Multimodal sentinel lymph node mapping with single-photon emission computed tomography (SPECT)/computed tomography (CT) and photoacoustic tomography. Transl. Res. 159, 175-181 (2012)

62. Krumholz, A., Shcherbakova, D.M., Xia, J., Wang, L.V. \& Verkhusha, V.V. Multicontrast photoacoustic in vivo imaging using near-infrared fluorescent proteins. Sci. Rep. 4, 3939 (2014).

63. Kim, J.W., Galanzha, E.I., Shashkov, E.V., Moon, H.M. \& Zharov, V.P. Golden carbon nanotubes as multimodal photoacoustic and photothermal high-contrast molecular agents. Nat. Nanotechnol. 4, 688-694 (2009).

64. Cox, B., Laufer, J.G., Arridge, S.R. \& Beard, P.C. Quantitative spectroscopic photoacoustic imaging: a review. J. Biomed. Opt. 17, 061202 (2012).

65. Tzoumas, S., Nunes, A., Deliolanis, N.C. \& Ntziachristos, V. Effects of multispectral excitation on the sensitivity of molecular optoacoustic imaging. J. Biophotonics 8, 629-637 (2015).

66. Taruttis, A., Morscher, S., Burton, N.C., Razansky, D. \& Ntziachristos, V. Fast multispectral optoacoustic tomography (MSOT) for dynamic imaging of pharmacokinetics and biodistribution in multiple organs. PLOS ONE 7, e30491 (2012).

67. Deliolanis, N.C. et al. Deep-tissue reporter-gene imaging with fluorescence and optoacoustic tomography: a performance overview. Mol. Imaging Biol. 16, 652-660 (2014).

68. Filonov, G.S. et al. Deep-tissue photoacoustic tomography of a genetically encoded near-infrared fluorescent probe. Angew. Chem. Int. Ed. Engl. 51, 1448-1451 (2012).
69. Galanzha, E.I. et al. Photoacoustic and photothermal cytometry using photoswitchable proteins and nanoparticles with ultrasharp resonances. J. Biophotonics 8, 81-93 (2013).

70. Jathoul, A.P. et al. Deep in vivo photoacoustic imaging of mammalian tissues using a tyrosinase-based genetic reporter. Nat. Photonics $\mathbf{9}$, 239-246 (2015).

71. Laufer, J., Jathoul, A., Pule, M. \& Beard, P. In vitro characterization of genetically expressed absorbing proteins using photoacoustic spectroscopy. Biomed. Opt. Express 4, 2477-2490 (2013).

72. Levi, J. et al. Design, synthesis, and imaging of an activatable photoacoustic probe. J. Am. Chem. Soc. 132, 11264-11269 (2010).

73. Laufer, J., Zhang, E., Raivich, G. \& Beard, P. Three-dimensional noninvasive imaging of the vasculature in the mouse brain using a high resolution photoacoustic scanner. Appl. Opt. 48, D299-D306 (2009)

74. Yao, J. et al. Noninvasive photoacoustic computed tomography of mouse brain metabolism in vivo. Neuroimage 64, 257-266 (2013).

75. Nasiriavanaki, M. et al. High-resolution photoacoustic tomography of resting-state functional connectivity in the mouse brain. Proc. Natl. Acad. Sci. USA 111, 21-26 (2014).

76. Jo, J. \& Yang, X. Functional photoacoustic imaging to observe regional brain activation induced by cocaine hydrochloride. J. Biomed. Opt. 16, 090506 (2011).

77. Liao, L.D. et al. Imaging brain hemodynamic changes during rat forepaw electrical stimulation using functional photoacoustic microscopy. Neuroimage 52, 562-570 (2010).

78. Pilatou, M.C., Marani, E., de Mul, F.F. \& Steenbergen, W. Photoacoustic imaging of brain perfusion on albino rats by using evans blue as contrast agent. Arch. Physiol. Biochem. 111, 389-397 (2003).

79. Yao, J. \& Wang, L.V. Photoacoustic brain imaging: from microscopic to macroscopic scales. Neurophotonics 1, 1877516 (2014).

80. Deng, Z.L., Wang, Z., Yang, X.Q., Luo, Q.M. \& Gong, H. In vivo imaging of hemodynamics and oxygen metabolism in acute focal cerebral ischemic rats with laser speckle imaging and functional photoacoustic microscopy. J. Biomed. Opt. 17, 081415 (2012).

81. Tsytsarev, V., Rao, B., Maslov, K.I., Li, L. \& Wang, L.V. Photoacoustic and optical coherence tomography of epilepsy with high temporal and spatial resolution and dual optical contrasts. J. Neurosci. Methods 216, 142-145 (2013).

82. Tang, J.B. et al. Noninvasive high-speed photoacoustic tomography of cerebral hemodynamics in awake-moving rats. J. Cereb. Blood Flow Metab. 35, 1224-1232 (2015)

83. Strohm, E.M., Moore, M.J. \& Kolios, M.C. Single cell photoacoustic microscopy: a review. IEEE J. Sel. Top. Quantum Electron. 22, 6801215 (2015)

84. Bost, W. et al. High frequency optoacoustic microscopy. Conf. Proc. IEEE Eng. Med. Biol. Soc. 2009, 5883-5886 (2009).

85. Strohm, E.M., Berndl, E.S.L. \& Kolios, M.C. Probing red blood cell morphology using high-frequency photoacoustics. Biophys. J. 105, 59-67 (2013).

86. Wang, L.D., Maslov, K. \& Wang, L.V. Single-cell label-free photoacoustic flowoxigraphy in vivo. Proc. Natl. Acad. Sci. USA 110, 5759-5764 (2013).

87. He, G., Xu, D., Qin, H., Yang, S. \& Xing, D. In vivo cell characteristic extraction and identification by photoacoustic flow cytography. Biomed. Opt. Express 6, 3748-3756 (2015).

88. Wilson, K.E., Wang, T.Y. \& Willmann, J.K. Acoustic and photoacoustic molecular imaging of cancer. J. Nucl. Med. 54, 1851-1854 (2013)

89. Herzog, E. et al. Optical imaging of cancer heterogeneity with multispectral optoacoustic tomography. Radiology 263, 461-468 (2012).

90. Laufer, J. et al. In vivo preclinical photoacoustic imaging of tumor vasculature development and therapy. J. Biomed. Opt. 17, 056016 (2012)

91. Staley, J. et al. Growth of melanoma brain tumors monitored by photoacoustic microscopy. J. Biomed. Opt. 15, 040510 (2010).

92. Brannon-Peppas, L. \& Blanchette, J.0. Nanoparticle and targeted systems for cancer therapy. Adv. Drug Deliv. Rev. 56, 1649-1659 (2004).

93. Mallidi, S., Luke, G.P. \& Emelianov, S. Photoacoustic imaging in cancer detection, diagnosis, and treatment guidance. Trends Biotechnol. 29 213-221 (2011)

94. Ray, A. et al. Targeted blue nanoparticles as photoacoustic contrast agent for brain tumor delineation. Nano Res. 4, 1163-1173 (2011).

95. Levi, J. et al. Molecular photoacoustic imaging of follicular thyroid carcinoma. Clin. Cancer Res. 19, 1494-1502 (2013). 
96. Yao, J., Maslov, K.I., Zhang, Y., Xia, Y. \& Wang, L.V. Label-free oxygen-metabolic photoacoustic microscopy in vivo. J. Biomed. Opt. 16, 076003 (2011).

97. Shao, Q. et al. In vivo photoacoustic lifetime imaging of tumor hypoxia in small animals. J. Biomed. Opt. 18, 076019 (2013).

98. Zhang, C., Maslov, K. \& Wang, L.V. Subwavelength-resolution label-free photoacoustic microscopy of optical absorption in vivo. Opt. Lett. 35, 3195-3197 (2010).

99. Xia, J. et al. Whole-body ring-shaped confocal photoacoustic computed tomography of small animals in vivo. J. Biomed. Opt. 17, 050506 (2012).

100. Erpelding, T.N. et al. Sentinel lymph nodes in the rat: noninvasive photoacoustic and US imaging with a clinical US system. Radiology 256, 102-110 (2010).

101. Taruttis, A. \& Ntziachristos, V. Advances in real-time multispectral optoacoustic imaging and its applications. Nat. Photonics 9, 219-227 (2015).
102. Daoudi, K. et al. Handheld probe integrating laser diode and ultrasound transducer array for ultrasound/photoacoustic dual modality imaging. Opt. Express 22, 26365-26374 (2014).

103. Allen, T.J. \& Beard, P.C. High power visible light emitting diodes as pulsed excitation sources for biomedical photoacoustics. Biomed. Opt. Express 7, 1260-1270 (2016).

104. Li, H., Dong, B., Zhang, Z., Zhang, H.F. \& Sun, C. A transparent broadband ultrasonic detector based on an optical micro-ring resonator for photoacoustic microscopy. Sci. Rep. 4, 4496 (2014).

105. Favazza, C.P., Guo, Z., Maslov, K. \& Wang, L.V. Optimal oblique light illumination for photoacoustic microscopy beyond the diffusion limit. In Photons Plus Ultrasound: Imaging and Sensing 2011 (Proceedings of the SPIE, 7899) 789900 (SPIE, 2011).

106. Cho, Y. et al. Handheld photoacoustic tomography probe built using optical-fiber parallel acoustic delay lines. J. Biomed. Opt. 19, 086007 (2014). 